\title{
Alcohol Withdrawal Syndrome
}

\author{
Rabindran ${ }^{1}$, Gedam DS ${ }^{2}$ \\ ${ }^{1}$ Dr. Rabindran, Consultant, Neonatologist, Billroth Hospital, Chennai, ${ }^{2}$ Dr D Sharad Gedam, Professor of Pediatrics, \\ L. N. Medical College, Bhopal, MP, India.
}

Address for Correspondence: Dr Rabindran, E mail: rabindranindia@ yahoo.co.in

\begin{abstract}
Alcohol withdrawal syndrome is a cluster of symptoms occurring when someone detoxes from alcohol. Alcohol dependence is one of the commonest psychiatric disorders, second only to major depression.
\end{abstract}

Keywords: Alcohol withdrawal, Delirium tremens, withdrawal seizures.

Alcohol withdrawal syndrome is a cluster of symptoms occuring when someone detoxes from alcohol. Alcohol dependence is one of the commonest psychiatric disorders, second only to major depression [1]. Approximately $50 \%$ of those with alcohol dependence experience withdrawal symptoms \& upto $10 \%$ experience delirium tremens [2]. Withdrawal symptoms usually start about 6 hours after alcohol cessation \& withdrawal seizure is frequently the first sign occuring within 6- 48 hours of alcohol cessation. According to American Academy of Family Physicians, timeline for withdrawal symptoms include 1) 6-12 hours after cessation- Minor hand tremors, Sleep disturbances, Low-level stress, anxiety, Stomach upset, loss of appetite, Sweating \& Headaches; 2) 12-48 hours after cessation - Hallucinations, Withdrawal seizures \& General tonic-clonic seizures; 3) 48-72 hours after cessation- Delirium tremens, Disorientation, Sweating, Increased heart rate, blood pressure \& temperature. Central adrenergic storm occuring during alcohol withdrawal results in hyperventilation, tachycardia, hypertension, tremor, hyperthermia \& diaphoresis. Low-grade fever occurs due to increased motor activity. Hypothermia occurs with Wernicke encephalopathy. The underlying pathophysiology of acute alcohol withdrawal is CNS hyperexcitation [3]. Chronic alcohol exposure leads to brain adaptation to effects of alcohol through changes in receptors- down-regulation of GABA receptor \& up-regulation of NMDA receptors leading to an increased tolerance, requiring higher blood alcohol levels to achieve similar effects of intoxication. After cessation of alcohol consumption, the GABA inhibitory effect is lost \& potentiation of
NMDA excitatory effect occurs leading to CNS hyperstimulation. Moreover in regular heavy drinkers, the body compensates for depressive effect of alcohol by increasing production of hormones \& brain chemicals such as serotonin, epinephrine \& dopamine which reaches abnormally high levels when a person abruptly stops drinking alcohol \& thereby causes hyperexcitation. Clinical diagnosis of alcohol withdrawal is based on 1) Clear evidence of recent alcohol cessation, 2) Symptoms cannot be attributed to any co-occurring medical disorders, 3) Significant decrease in functioning in socio-occupational areas due to withdrawal symptoms. Complete blood count, Comprehensive metabolic panel, CT Scan, Lipase and Urinalysis, Serum pH \&osmolality, ECG, Serum salicylate, CXR, Coagulation panel (PT/INR, PTT) are done to evaluate such patients. Serum ethanol levels only reveal recent alcohol consumption, not chronic alcohol intake which predisposes to withdrawal [4]. Mean corpuscular volume, serum $\gamma$-glutamyl transpeptidase \& carbohydrate-deficient transferrin are widely studied with variable predictive value [5]. Metabolic complications of alcohol withdrawal include Alcoholic ketoacidosis, Electrolyte disorders (eg, hypomagnesemia, hypokalemia, hypernatremia) \& Vitamin deficiencies (eg, thiamine, phytonadione, cyanocobalamin, folic acid). Cardiac complications include Takotsubo cardiomyopathy. Gastrointestinal complications include Pancreatitis, Gastrointestinal bleeding (eg, peptic ulcer, esophageal varices, gastritis) \& Hepatic cirrhosis. Infectious complications include Pneumonia, Meningitis \& Cellulitis. Neurologic complications include Wernicke-Korsakoff syndrome, 
Cerebral atrophy, Cerebellar degeneration, Subdural or epidural haemorrhage \& Peripheral neuropathy. Delirium tremens (DT) is a rapid-onset, fluctuating disturbance of attention \& cognition along with alcohol withdrawal symptoms \& autonomic instability [6]. It occurs in $3-5 \%$ of patients who are hospitalized for alcohol withdrawal [7]. It usually begins 3 days after the appearance of withdrawal symptoms \& lasts for 1-8 days. DT can be predicted by factors like History of previous DT or sustained drinking, CIWA scores > $15, \mathrm{SBP}>150, \mathrm{HR}>100$, Recent or prior withdrawal seizures, Recent misuse of other depressants \& Concomitant medical problems. Medically supervised medication for detox includes Benzodiazepines (eg. diazepam, chlordiazepoxide, lorazepam, Oxazepam), anti-seizures drugs \& Beta-blockers [1]. Decision to give benzodiazepines is often based on symptomtriggered therapy, as evaluated by Clinical Institute Withdrawal Assessment for Alcohol (CIWA) scale. Phenobarbitone reduces symptoms by producing a generalized decrease in neurotransmission. Acamprosate, Naltrexone, Nalmefene \& Baclofen helps to ease alcohol cravings. Disulfiram produces unpleasant symptoms like flushing, vomiting, palpitations \& headache when alcohol is taken. Magnesium protects against seizures \& arrhythmias. Clonidine due to its central alpha ${ }_{2}$ - agonist activity reduces central output of adrenergic neurotransmitters $\&$ improves aberrant vital signs. Propranolol decreases blood pressure, pulse rate \& tremor. Replenishing vitamins can prevent Wernicke-Korsakoff syndrome (with thiamine), correct megaloblastic anemia (with folic acid and cyanocobalamin), correct high-output CHF (with thiamine) \& halt peripheral neuropathy (with cyanocobalamin). Many patients have multiple management issues (withdrawal symptoms, delirium tremens, Wernicke-Korsak off syndrome, seizures, depression, polysubstance abuse, electrolyte disturbances \& liver disease) which require a coordinated, multidisciplinary approach. Hence prompt monitoring with timely management is required to overcome alcohol withdrawal syndrome. Anil Kumar et al in this issue concluded that SD is highly prevalent in patients with alcohol dependence and all domains of sexual functioning are affected. Hence, the clinicians should routinely enquire about SD and do motivational counselling for patients to abstain from alcohol use. [8]

Funding: Nil, Conflict of interest: None initiated, Permission from IRB: Yes

\section{Reference:}

1. Sachdeva A, Choudhary M, Chandra M.Alcohol Withdrawal Syndrome: Benzodiazepines and Beyond. J Clin Diagn Res. 2015 Sep;9(9): VE01-VE07. doi: 10. 7860 / JCDR/2015/13407.6538.

2. Melson J, Kane M, Mooney R, Mcwilliams J, Horton T5. Improving alcohol withdrawal outcomes in acute care. Perm J. 2014 Spring;18(2):e141-5. doi: 10.7812 /TPP/13-099.

3. Yanta JH, Swartzentruber GS, Pizon AF. Alcohol withdrawal syndrome: Improving outcomes through early identification and aggressive treatment strategies. Emerg Med Pract. 2015 Jun;17(6):1-18; quiz 19.

4. Sharp B, Schermer CR, Esposito TJ, Omi EC, TonThat H, Santaniello JM. Alcohol Withdrawal Syndrome in Trauma Patients: A Prospective Cohort Study. J Trauma Treat 2012;1(4):1:128. doi:10.4172/2167-1222. 1000128.

5.Hashimoto E, Riederer PF, Hesselbrock VM, Hesselbrock MN, Mann K, Ukai W, Sohma H, Thibaut F, Schuckit MA, Saito T. Consensus paper of the WFSBP task force on biological markers: biological markers for alcoholism. World J Biol Psychiatry. 2013 Dec;14(8):549-64.doi:10.3109/15622975.2013. 838302.

6. Schuckit Marc A. Recognition and Management of Withdrawal Delirium (Delirium Tremens). N Engl J Med 2014; 371:2109-2113November 27, 2014DOI: 10. 1056/ NEJMra1407298.

7. Mainerova B, Prasko J, Latalova K, Axmann K, Cerna M, Horacek R, Bradacova R. Alcohol withdrawal delirium - diagnosis, course and treatment. Biomed Pap Med Fac Univ Palacky Olomouc Czech Repub. 2015 Mar;159(1):44-52. doi: 10.5507/bp.2013.089.

8. Anil Kumar B.N, Shalini M, Sanjay Raj G, Prasannakumar D.R. Prevalence, typology and clinical correlates of sexual dysfunction among men with alcohol dependence syndrome. Int J Med Res Rev 2016;4(10):1826-1832.doi:10.17511/ijmrr. 2016.i10.19.

\section{How to cite this article?}

Rabindran, Gedam DS. Alcohol Withdrawal Syndrome. Int J Med Res Rev 2016;4(10):1722-1723.doi:10.17511/ijmrr. 2016.i10.01. 